\title{
Exploiting Accelerometers to Improve Movement Classification for Prosthetics
}

\author{
Arjan Gijsberts \\ Idiap Research Institute \\ 1920 Martigny, Switzerland \\ Email: arjan.gijsberts@idiap.ch
}

\author{
Barbara Caputo \\ Idiap Research Institute \\ 1920 Martigny, Switzerland \\ Email: bcaputo@idiap.ch
}

\begin{abstract}
Recent studies have explored the integration of additional input modalities to improve myoelectric control of prostheses. Arm dynamics in particular are an interesting option, as these can be measured easily by means of accelerometers. In this work, the benefit of accelerometer signals is demonstrated on a large scale movement classification task, consisting of 40 hand and wrist movements obtained from 20 subjects. The results demonstrate that the accelerometer modality is indeed highly informative and even outperforms surface electromyography in terms of classification accuracy. The highest accuracy, however, is obtained when both modalities are integrated in a multi-modal classifier.
\end{abstract}

\section{INTRODUCTION}

Externally powered upper-limb prostheses are almost exclusively controlled using one or more surface electromyography (sEMG) signals. Their functionality has remained limited to a small number of degrees of freedom, despite several decades of experience and considerable research efforts. This slow progress is to large extent due to the nature of the sEMG signal, which is characterized by a low voltage amplitude, broad bandwidth, and sensitivity to sensor placement and muscle fatigue. All of these make its use to control the low frequency activity of a prosthetic hand less than straightforward. Furthermore, even some intact subjects have difficulties to produce sEMG signals that are sufficiently precise for dexterous or proportional control [1].

Recent studies have therefore tried to complement or replace the sEMG signals with additional input modalities, such as myokinemetry (muscle bulge) [2], ultrasound [3], [4], and accelerometry [5]. The latter modality is particularly interesting, since accelerometers are available at low cost and can easily be integrated in the prosthetic socket or casing. Moreover, a study on the relation between sEMG and accelerometry revealed that the two modalities appear to capture different aspects of movement and are thus best considered complementary [6]. This synergy was recently confirmed by Fougner, Scheme, Chan, et al. [5], who used Linear Discriminant Analysis (LDA) to classify eight wrist and hand movements acquired in five different limb positions. Their experiments using 10 subjects demonstrate that it is preferable to add an accelerometer to a two-site sEMG system rather than a third electrode. Related to the context of prosthetic control, the integration of accelerometers and sEMG has also proven useful for sign language recognition [7], [8]. For example, the information fusion framework proposed by Zhang, Chen, Li, et al. [8] classified 72 Chinese sign language words with more than $95 \%$ accuracy, largely due to the complementary nature of both modalities.

The present work continues the investigation on the use of accelerometers for prosthetic control. In contrast to prior work by Fougner, Scheme, Chan, et al. [5], we consider 40 different movements acquired from 20 subjects. Furthermore, three types of sEMG features are evaluated, both individually as well as jointly, while a non-linear kernel method is used as classifier. These more advanced methods are intended to eliminate the possibility that improvements when including the accelerometer modality are merely due to suboptimal use of the original sEMG modality. As an additional result of the experiments, we also report whether it is beneficial to combine multiple sEMG features, as compared to using the best individual feature type.

The remainder of this paper is organized as follows. The data acquisition, movements, and preprocessing steps are described in Section II. Subsequently, the feature extraction methods and the classifier are detailed in Section III, which also includes a description of the methodology used for the classification experiments. The experimental results and discussion thereof are presented in Section IV. Finally, Section V contains concluding remarks as well as pointers to future work.

\section{DATABASE}

The database used in this work is the second iteration of the database acquired as part of the Non-Invasive Adaptive Prosthetics (NinaPro) project, which aims to support research on sEMG-based control by means of a large-scale and publicly available database [9]. Noteworthy changes in the acquisition procedure with respect to the earlier published variant of the database are (1) the use of different electrodes that allow recording raw sEMG signals, (2) removal of the single digit movements, and (3) a reduction in the number of repetitions to reduce muscle fatigue and cognitive load. A concise description of the acquisition and preprocessing procedure follows, based on the relevance to this present work. The interested reader is referred to the work by Atzori, Gijsberts, Heynen, et al. [9] for a more thorough overview.

\section{A. Acquisition}

The data has been acquired using a Delsys ${ }^{\mathrm{TM}}$ Trigno Wireless System $(\mathbb{R}$. This system consists of a base station and multiple wireless electrodes, each of which contains an sEMG sensor sampled at $2 \mathrm{kHz}$ as well as a 3 -axes accelerometer at 


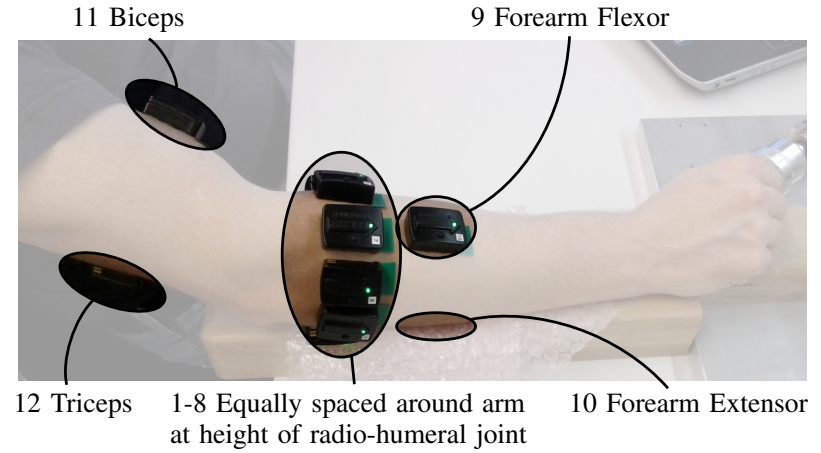

Fig. 1. Placement of the 12 electrodes on the arm. The electrode on the forearm extensor is occluded by the arm and therefore not visible in this image.

$146 \mathrm{~Hz}$. The base station receives the sEMG and accelerometer streams over a proprietary wireless communication protocol and relays these via a standard USB connection to the laptop responsible for data acquisition. Twelve electrodes were attached to the subject's arm according to the configuration illustrated in Figure 1. The rationale behind this exact placement is to combine dense sampling of the forearm (electrodes $1-8$ ) with additional electrodes that are targeted at specific muscles (electrodes $9-12$ ).

A total of 20 intact subjects participated in the data acquisition, with an age distribution of $30.4 \pm 4.5 \mathrm{y}$ and consisting of 14 male and 6 female participants. During the acquisition, each subject was seated at a desk and instructed to perform the movements that were demonstrated by means of a video on a computer screen. In this manner, the subjects performed 6 consecutive repetitions of the 40 movements described in Table I, where each repetition was alternated with a rest posture of similar duration. After the first 17 hand and wrist movements, subjects were allowed to rest for some minutes before proceeding with the remaining 23 grasp and functional movements.

\section{B. Data Preprocessing}

Several offline preprocessing steps have been performed to prepare the data for the classification experiments. The sEMG signals were first filtered to remove $50 \mathrm{~Hz}$ power-line interference (and its harmonics) using a Hampel filter [10]. Subsequently, the raw accelerometer ${ }^{1}$ and stimulus (i.e., the movement label) signals were synchronized with the sEMG signal by upsampling them using linear interpolation to the $2 \mathrm{kHz}$ sampling rate of the latter.

A problem with the described acquisition procedure is that the movements performed by the subjects may not match perfectly with the video stimulus. On several occasions, a subject would start the actual movement slightly after the start of the video and finish the movement either in advance or with some delay. This misalignment between the stimulus and the actual movement can be attributed to human reaction times as well as our explicit instruction to perform natural movements rather than exactly copying the kinematics of the

\footnotetext{
${ }^{1}$ No processing was applied other than the standard processing onboard the Trigno electrodes.
}

TABLE I. DESCRIPTION OF THE 40 MOVEMENTS.

\begin{tabular}{|c|c|c|}
\hline & \# & Description \\
\hline 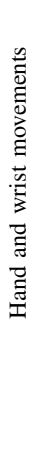 & $\begin{array}{r}1 \\
2 \\
3 \\
4 \\
5 \\
6 \\
7 \\
8 \\
9-10 \\
11-12 \\
\\
\\
13-14 \\
15-16 \\
17\end{array}$ & $\begin{array}{l}\text { Thumb up } \\
\text { Extension of index and middle finger while flexing } \\
\text { others (cf. "V-sign") } \\
\text { Flexion of ring and little finger while extending others } \\
\text { Thumb opposing base of little finger } \\
\text { Abduction of the fingers } \\
\text { Fingers flexed together in fist } \\
\text { Pointing index } \\
\text { Adduction of extended fingers } \\
\text { Wrist supination and pronation (rotation axis through the } \\
\text { middle finger) } \\
\text { Wrist supination and pronation (rotation axis through the } \\
\text { little finger) } \\
\text { Wrist flexion and extension } \\
\text { Wrist radial and ulnar deviation } \\
\text { Wrist extension with closed hand }\end{array}$ \\
\hline 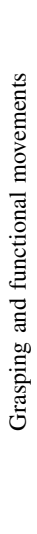 & $\begin{array}{r}18-19 \\
20 \\
21 \\
22 \\
23 \\
24 \\
25 \\
26 \\
27-29 \\
30 \\
31-32 \\
33 \\
34 \\
35 \\
36 \\
37 \\
38 \\
39 \\
40\end{array}$ & $\begin{array}{l}\text { Large and small diameter grasp } \\
\text { Fixed hook grasp } \\
\text { Index finger extension grasp } \\
\text { Medium wrap } \\
\text { Ring grasp } \\
\text { Prismatic four fingers grasp } \\
\text { Stick grasp } \\
\text { Writing tripod grasp } \\
\text { Power, three finger, and precision sphere grasp } \\
\text { Tripod grasp } \\
\text { Prismatic and tip pinch grasp } \\
\text { Quadpod grasp } \\
\text { Lateral grasp } \\
\text { Parallel extension grasp } \\
\text { Extension type grasp } \\
\text { Power disk grasp } \\
\text { Open a bottle with a tripod grasp } \\
\text { Turn a screw } \\
\text { Cut something }\end{array}$ \\
\hline
\end{tabular}

video stimulus. The resulting erroneous movement labels have been corrected using a Generalized Likelihood Ratio approach [11], which realigns the movement labels to time windows that contain increased sEMG activity.

\section{EXPERIMENTAL SETUP}

Per the control scheme proposed by Englehart and Hudgins [12], the sEMG signals were segmented by means of a sliding window prior to feature extraction. A window length of $400 \mathrm{~ms}$ (800 samples) has been used, based on the results of Kuzborskij, Gijsberts, and Caputo [11] as well as additional preliminary experiments. The increment of the sliding window was set to $10 \mathrm{~ms}$ (20 samples), such that the overlap between successive windows is $97.5 \%$.

The data for each subject was subsequently split into training and test sets based on repetitions: the first, third, fourth, and sixth repetition were used for training, while the test set contained the second and fifth repetition for each movement. All channels were standardized to have a zero mean and unit standard deviation, based on statistics calculated solely on data from the training set. Note that this scaling was applied prior to segmenting the signals into windows. For computational reasons, the size of the training and hyperparameter optimization sets was reduced by increasing the increment of the sliding window to $100 \mathrm{~ms}$ and $400 \mathrm{~ms}$, respectively. The resulting training and test set contain approximately 15000 and 70000 windows for each of the subjects. 
TABLE II. FEATURE CONFIGURATIONS.

\begin{tabular}{llcll}
\hline Name & Modality & Window & Configuration & Kernel \\
\hline RMS & sEMG & $400 \mathrm{~ms}$ & & exp- $\chi^{2}$ \\
mDWT & sEMG & $400 \mathrm{~ms}$ & db7 wavelet, 3 levels & exp- $\chi^{2}$ \\
HIST & sEMG & $400 \mathrm{~ms}$ & 20 bins, $3 \sigma$ threshold & exp- $\chi^{2}$ \\
MEAN & ACC & $400 \mathrm{~ms}$ & & RBF \\
\hline
\end{tabular}

\section{A. Features}

Perhaps the most commonly used feature representation in the sEMG literature is the relatively simple Root Mean Square (RMS) of the signal. A compelling argument for using this feature is that (under ideal conditions) there is a quasi-linear relationship between the RMS value and the force exerted by a muscle. Furthermore, this feature type is easily implemented both in digital as well as analog systems.

A more advanced alternative that has recently gained popularity is the Discrete Wavelet Transform (DWT). This transformation decomposes the signal in terms of a basis function (i.e., the wavelet) at different levels of resolution, resulting in a high-dimensional frequency-time representation. Lucas, Gaufriau, Pascual, et al. [13], however, have demonstrated that for sEMG-based classification it is sufficient to preserve only the marginals at each level of the decomposition, thereby drastically reducing the dimensionality of the feature representation. Henceforth, this variant will be referred to as marginal Discrete Wavelet Transform (mDWT). Although a variety of wavelet functions have been used in the context of sEMG [14], preliminary experiments on our data revealed that the $7^{\text {th }}$ order Daubechies wavelet performed slightly better than others in a small pool of candidate functions. The marginal coefficients up to the third level obtained with this wavelet function have therefore been used in the experimental validation.

The third considered feature type is the sEMG Histogram (HIST) [15], which computes a histogram within the analysis window given a predefined number of bins. Despite its simplicity, the HIST feature has demonstrated excellent performance for sEMG-based movement classification [11], [15]. Rather than setting the lower and upper thresholds based on the extrema of the signal, we exploited the fact that the sEMG signals were standardized and set the thresholds to three standard deviations. In addition, outliers were captured in "extremal" bins on each side, such that the bin edges become $[-\infty,-3, \ldots,+3, \infty]$. The total number of bins was fixed at 20.

Following the approach by Fougner, Scheme, Chan, et al. [5], the mean value (MEAN) within the processing window is used as feature for the accelerometer (ACC) modality. The dense placement of the electrodes (see Figure 1) suggests that many of the ACC channels will be highly correlated. However, this redundancy is not expected to affect overall performance and all 36 channels ( 12 electrodes $\times 3$ axes) have been used in the following experiments. An overview of all the considered features and the corresponding configuration is presented in Table II.

\section{B. Classifier}

Classification of the movement labels from the described features and modalities was done using the Kernel Regularized
Least Squares (KRLS) classifier [16]. This kernel method is closely related to the well-known Support Vector Machine (SVM) [17], but offers the advantage that training consists of solving a linear system of equations. This allows multiple output dimensions to be learned simultaneously at negligible additional cost. This latter is relevant, since the multiclass problem under consideration here is reduced to 41 binary classification problems (i.e., 40 movements and rest) using the well-known one-versus-all reduction.

The most commonly used kernel function is the Radial Basis Function (RBF) kernel

$$
k(\boldsymbol{x}, \boldsymbol{y})=\exp \left(-\gamma\|\boldsymbol{x}-\boldsymbol{y}\|^{2}\right) \quad \text { for } \gamma>0,
$$

which has demonstrated excellent performance in a large variety of application domains. However, the exp- $\chi^{2}$ kernel

$$
k(\boldsymbol{x}, \boldsymbol{y})=\exp \left(-\gamma \sum_{i=1}^{n} \frac{\left(x_{i}-y_{i}\right)^{2}}{x_{i}+y_{i}}\right) \text { for } \gamma>0,
$$

may be more appropriate for histogram-like feature representations. Interestingly, during preliminary experiments we found that the exp- $\chi^{2}$ kernel outperformed the RBF kernel for all considered sEMG features. For the MEAN features over the ACC modality, on the other hand, the standard RBF kernel was used.

The formulation of the KRLS classifier includes a regularization parameter $\lambda$, which balances the tradeoff between under- and overfitting. This parameter was tuned together with the kernel parameter $\gamma$ using 4-fold cross validation, where each of the folds corresponds to one of the 4 training repetitions. This cross validation strategy ensures that the differences in distribution between different repetitions were taken into account when optimizing the hyperparameters. In order to increase the likelihood of finding a (nearly) optimal configuration, parameters were selected using a dense grid search with $\lambda \in\left\{2^{-16}, 2^{-15}, \ldots, 2^{2}, 2^{3}\right\}$ and $\gamma \in\left\{2^{-20}, 2^{-19}, \ldots, 2^{2}, 2^{3}\right\}$.

Combining multiple features or modalities is typically implemented with linear methods by concatenating the individual feature vectors (e.g., [5]). In kernel-based methods, however, it is more suitable to add the kernel functions instead, since this corresponds to concatenating the hypothetical feature spaces induced by the respective kernels. Recently proposed methods for multi kernel learning (MKL) allow to optimize the contribution of each kernel as well (see [18] and references therein). Nevertheless, the average of the kernels was used in this work for simplicity reasons. Moreover, related work in computer vision has demonstrated that kernel averaging performs almost as good when using a relatively small number of informative features [19], as is the case in this study.

\section{RESUlTS}

The aim of this paper is to investigate how informative the accelerometer modality is for large-scale movement classification, especially in the context of prosthetic control and thus when used in combination with sEMG signals. The benefit of the accelerometer modality will be measured in terms of the improvement in classification accuracy as compared to standard sEMG-only classifiers. 


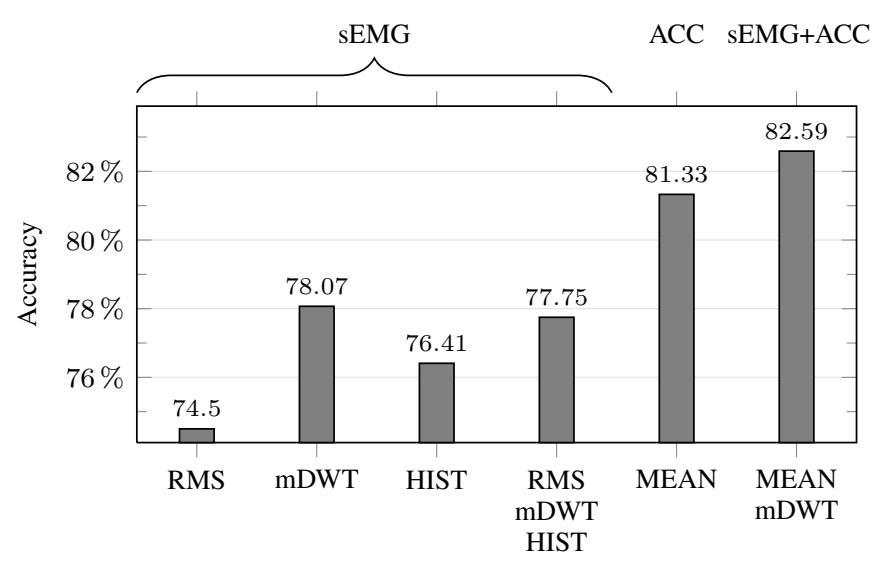

Fig. 2. Average classification accuracy over the 20 subjects when using (from left to right) the three sEMG based features individually and when combining these, the MEAN features of the ACC modality, and when combining the sEMG and ACC modalities.

\section{A. Single Modality Classifiers}

The classification accuracy for the three individual sEMG features lies approximately between $74 \%$ and $78 \%$, as shown on the left hand side of Figure 2. In contrast to the study by Kuzborskij, Gijsberts, and Caputo [11], there is a significant advantage in using the higher dimensional $\mathrm{mDWT}$ and HIST features as compared to RMS features ${ }^{2}$ ( $p \ll 1 \%$, sign test). Furthermore, Figure 2 also shows that combining all three sEMG feature types in a single classifier results in a slight decrease in accuracy $(p \leq 1.2 \%)$, suggesting that the HIST and RMS features may not contain additional information that is not preserved by the mDWT features as well. Interestingly, the accuracy of the MEAN features over the accelerometer modality is more than $81 \%$ and thus significantly higher than any of the considered features of the sEMG signal $(p \ll 1 \%)$. This surprisingly high classification accuracy confirms that arm dynamics are highly informative for movement classification.

\section{B. Combining Multiple Modalities}

The high performance of the ACC-based classifier raises the question whether the accuracy can be further improved by integrating both ACC and sEMG modalities. In Figure 2, we observe that this is indeed the case, as the multi-modal mDWT+MEAN classifier attains a significant improvement of $1.26 \%$ over the ACC-only classifier $(p \leq 2.6 \%$ ). This proves empirically that the ACC and sEMG modalities can be considered as complementary, a result that is in line with related studies.

Figure 3 demonstrates that inclusion of the ACC modality uniformly increases the classification accuracy for all 40 (nonrest) movements. This improved accuracy is to large extent achieved by reducing the misclassifications of actual movements as rest, as is evident from the large number of blue elements in the first column of Figure 3. However, we also observe some blue clusters around the diagonal, which indicate that the ACC modality also helps to disambiguate certain groups of related movements.

\footnotetext{
${ }^{2}$ This can most likely be attributed to the availability of raw sEMG signals in the present work.
}

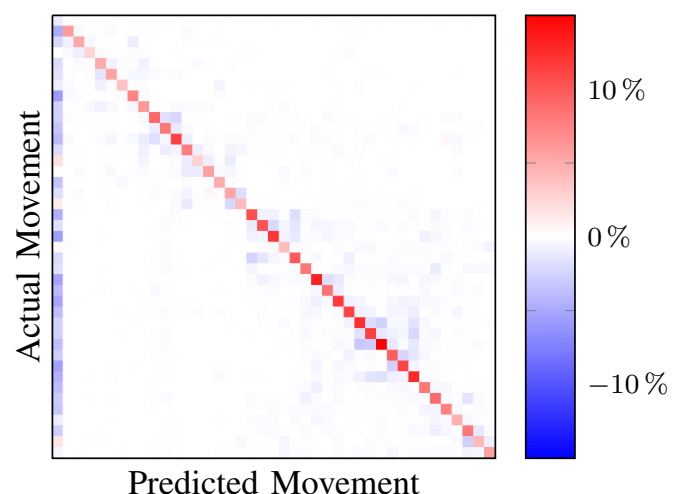

Fig. 3. Difference between the confusion matrices of the mDWT and the mDWT+MEAN classifiers (see text). The leftmost column and the top row correspond to the rest posture, while the remaining columns and rows follow the order described in Table I. Red elements on the diagonal indicate an improvement in correct classifications, whereas blue elements off the diagonal indicate a reduction of misclassifications. This figure is best viewed in color.

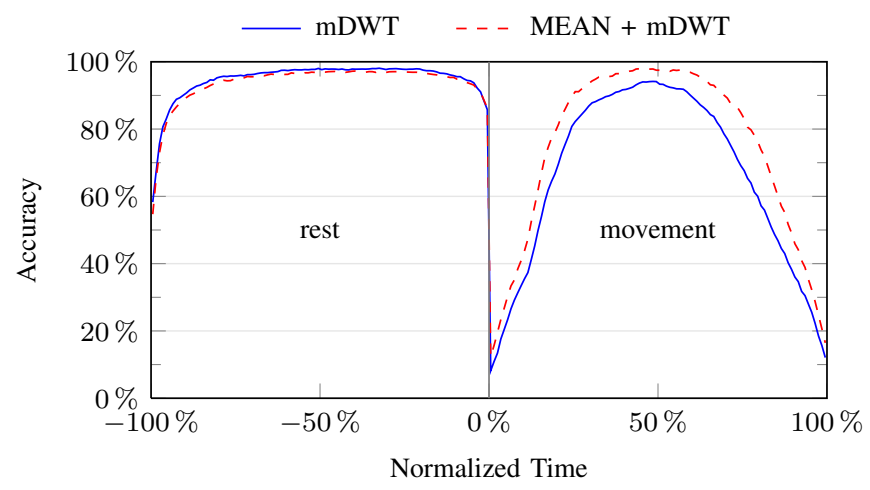

Fig. 4. Accuracy with respect to normalized time of the movements and the preceding rest for the mDWT and the mDWT+MEAN classifiers. The reported accuracy is averaged over all movements and subjects.

The improved accuracy is not only consistent over movement classes, but also throughout the entire duration of the movements (see Figure 4). This figure also gives more insight into the actual distribution of the misclassifications, which are concentrated during the movement on- and offset. This is not surprising, since movements are continuous trajectories that transition gradually from one type to another, while the discrete movement labels change abruptly between classes. Consequently, the accuracy drops drastically when the label changes from rest to movement and vice versa, since the input modality (i.e., the "evidence") does not yet support strong predictions of movement change. Misclassifications during these phases are best characterized as a delay in predicting the correct class, rather than incorrectly classifying one movement as another. In fact, when we restrict our attention to the center of the movement (i.e., around the $50 \%$ point on the $\mathrm{x}$-axis in Figure 4), then the multi-modal mDWT+MEAN classifier achieves an impressive accuracy of nearly $98 \%$.

\section{CONCLUSIONS}

The present work investigated the use of accelerometers for large-scale movement classification. Experiments on a data set of 40 hand and wrist movements acquired from 20 
subjects revealed that the accelerometer modality outperformed the sEMG modality. However, a multi-modal classifier that integrated both modalities improved over either uni-modal classifier, achieving an overall accuracy of almost $83 \%$. When concentrating solely on the center of the movement trajectory, this classifier attained an accuracy of nearly $98 \%$. These results indicate that the inclusion of accelerometers can be a costeffective approach to improve the dexterity of myoelectric prostheses.

The integration of multiple sEMG-based features caused a slight degradation of accuracy when compared to the best performing single feature type. This might indicate that the integration by means of kernel averaging performs suboptimally. In future work, it would be interesting to investigate whether further gains can be made by employing advanced MKL methods that automatically optimize the contribution of each feature type or modality.

Finally, a shortcoming in the current study is that the data was acquired from intact subjects. It is well-known that amputees typically produce lower quality sEMG signals, primarily due to a reduced use of the muscles and possible damage caused by trauma or the amputation procedure. However, it is plausible that the improvements will be even more significant in that case, since the lower arm dynamics, as measured by the accelerometers, seem less likely to be impacted by the amputation. Future work will thus concentrate on validating the current findings with actual amputees.

\section{ACKNOWLEDGMENTS}

The authors would like to thank all subjects that participated in the data acquisitions. This work is partially supported by the Swiss National Science Foundation Sinergia project NinaPro (http://www.idiap.ch/project/ninapro/).

\section{REFERENCES}

[1] H. Bouwsema, C. K. van der Sluis, and R. M. Bongers, "Learning to control opening and closing a myoelectric hand," Archives of Physical Medicine and Rehabilitation, vol. 91, no. 9, pp. 1442-1446, 2010.

[2] L. P. J. Kenney, I. Lisitsa, P. Bowker, G. H. Heath, and D. Howard, "Dimensional change in muscle as a control signal for powered upper limb prostheses: a pilot study," Medical Engineering \& Physics, vol. 21, no. 8, pp. 589-597, 1999.

[3] X. Chen, S. Chen, and G. Dan, "Control of powered prosthetic hand using multidimensional ultrasound signals: a pilot study," in Proceedings of the 6th International Conference on Universal Access in HumanComputer Interaction (UAHCI): Applications and Services - Volume Part IV, 2011, pp. 322-327.

[4] C. Castellini, G. Passig, and E. Zarka, "Using ultrasound images of the forearm to predict finger positions," IEEE Transactions on Neural Systems and Rehabilitation Engineering, vol. 20, no. 6, pp. 788-797, 2012.

[5] A. Fougner, E. Scheme, A. D. C. Chan, K. Englehart, and $\varnothing$. Stavdahl, "A multi-modal approach for hand motion classification using surface emg and accelerometers," in Annual International Conference of the IEEE Engineering in Medicine and Biology Society (EMBC), 2011, pp. 4247-4250.
[6] A. Keil, T. Elbert, and E. Taub, "Relation of accelerometer and emg recordings for the measurement of upper extremity movement," Journal of Psychophysiology, vol. 13, no. 2, p. 77, 1999.

[7] J. Kim, J. Wagner, M. Rehm, and E. André, "Bi-channel sensor fusion for automatic sign language recognition," in IEEE International Conference on Automatic Face and Gesture Recognition, Sep. 2008, pp. 1-6.

[8] X. Zhang, X. Chen, Y. Li, V. Lantz, K. Wang, and J. Yang, "A framework for hand gesture recognition based on accelerometer and emg sensors," IEEE Transactions on Systems, Man, and Cybernetics, Part A: Systems and Humans, vol. 41, no. 6, pp. 1064-1076, Nov. 2011.

[9] M. Atzori, A. Gijsberts, S. Heynen, A.-G. M. Hager, O. Deriaz, P. van der Smagt, C. Castellini, B. Caputo, and H. Müller, "Building the Ninapro database: a resource for the biorobotics community," in Proceedings of IEEE International Conference on Biomedical Robotics and Biomechatronics (BioRob), 2012, pp. 1258-1265.

[10] D. P. Allen, "A frequency domain hampel filter for blind rejection of sinusoidal interference from electromyograms," Journal of Neuroscience Methods, vol. 177, no. 2, pp. 303-310, 2009.

[11] I. Kuzborskij, A. Gijsberts, and B. Caputo, "On the challenge of classifying 52 hand movements from surface electromyography," in Annual International Conference of the IEEE Engineering in Medicine and Biology Society (EMBC), 2012, pp. 4931-4937.

[12] K. Englehart and B. Hudgins, "A robust, real-time control scheme for multifunction myoelectric control," IEEE Transactions on Biomedical Engineering, vol. 50, no. 7, pp. 848-854, 2003.

[13] M.-F. Lucas, A. Gaufriau, S. Pascual, C. Doncarli, and D. Farina, "Multi-channel surface EMG classification using support vector machines and signal-based wavelet optimization," Biomedical Signal Processing and Control, vol. 3, no. 2, pp. 169-174, Apr. 2008.

[14] J. Rafiee, M. A. Rafiee, N. Prause, and M. P. Schoen, "Wavelet basis functions in biomedical signal processing," Expert Systems with Applications, vol. 38, no. 5, pp. 6190-6201, May 2011.

[15] M. Zardoshti-Kermani, B. C. Wheeler, K. Badie, and R. M. Hashemi, "EMG feature evaluation for movement control of upper extremity prostheses," IEEE Transactions on Rehabilitation Engineering, vol. 3, no. 4, pp. 324-333, 1995.

[16] R. Rifkin, G. Yeo, and T. Poggio, "Regularized least squares classification," in Advances in Learning Theory: Methods, Model and Applications, vol. 190, VIOS Press, 2003, ch. 7, pp. 131-154.

[17] N. Cristianini and J. Shawe-Taylor, An Introduction to Support Vector Machines and Other Kernel-based Learning Methods. Cambridge University Press, 2000.

[18] F. Orabona, J. Luo, and B. Caputo, "Multi kernel learning with online-batch optimization," Journal of Machine Learning Research, vol. 13, pp. 227-253, 2012.

[19] P. V. Gehler and S. Nowozin, "On feature combination for multiclass object classification," in IEEE International Conference on Computer Vision (ICCV), 2009, pp. 221-228. 